\title{
Hilfe bei hepatischer Enzephalopathie
}

\section{Mit dem nicht resorbierbaren Anti- biotikum Rifaximin gelingt bei über $80 \%$ der Leberzirrhose-Patienten mit MELD-Scores über 20 auch nach einem Jahr noch eine Remission der hepatischen Enzephalopathie.}

- Die hepatischen Enzephalopathie ist eine schwerwiegende neuropsychiatrische Komplikation der Leberzirrhose, die bei 50-70 \% der Zirrhose-Patienten beobachtet wird. Man nimmt an, dass eine Anhäufung von Toxinen, wie zum Beispiel Ammoniak, dafür verantwortlich ist.

Die seit Jahrzehnten gebräuchliche Standardtherapie besteht in der Verabreichung von Lactulose zur Beschleunigung der Darmpassage, wodurch die Resorption von Ammoniak verringert werden soll. Diese Therapie ist zum einen wenig effektiv, verursacht zum anderen auch unangenehme gastrointestinale Nebenwirkungen, sodass eine Langzeittherapie kaum toleriert wird. Ein alternativer Weg ist die Elimination von Darmbakterien durch nicht resorbierbare Antibiotika.

Rifaximin, das bisher hauptsächlich zur Behandlung der Reisediarrhö Anwendung fand, hat in zwei kontrollierten Studien seine Überlegenheit gegenüber

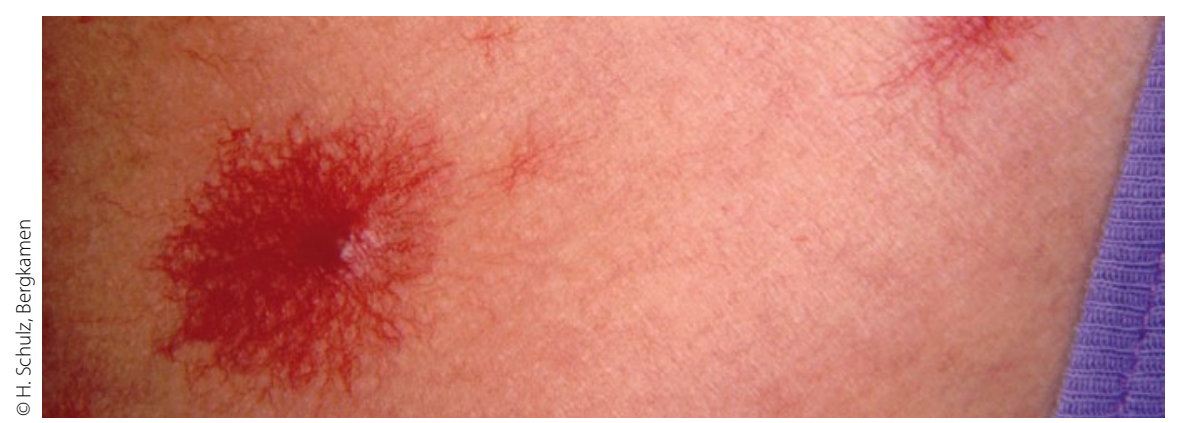

Naevi aranei bei alkoholtoxischer Leberzirrhose, w 42, Halsausschnitt.

nicht resorbierbaren Disacchariden zur Behandlung der hepatischen Enzephalopathie bewiesen.

In einer retrospektiven Auswertung der Krankenakten von Patienten, die zwischen 2004 und 2009 wegen einer Leberzirrhose mit hepatischer Enzephalopathie in fünf Zentren behandelt wurden, fand man 149 Patienten unter langfristiger Rifaximin-Monotherapie (400$1600 \mathrm{mg} / \mathrm{Tag}$ ) und 54 unter einer Kombinationstherapie aus Rifaximin und Lactulose (90 ml/Tag). $81 \%$ der Patienten unter Monotherapie und $67 \%$ unter Kombinationstherapie befanden sich nach einem Jahr in Remission der Enzephalopathie. Rezidive der Enzephalopathie traten in der Monotherapie-Gruppe nur zu 19\%, in der Kombinationstherapiegruppe zu 33\% auf. Aus diesen Daten ist allerdings nicht zu schließen, dass die Monotherapie effektiver ist als die Kombinationstherapie. Vielmehr dürften Patienten mit schwerer Enzephalopathie primär bereits in der Kombination behandelt worden sein.

\section{Kommentar}

Auch wenn es sich um eine retrospektive Studie mit relativ kleiner Patientenzahl handelt, so mehren sich doch die Hinweise, dass Rifaximin ein erfolgreiches und vor allem gut verträgliches Therapieprinzip auch zur langfristigen Behandlung der hepatischen Enzephalopathie darstellt.

H. S. FüESSL *

- Neff G. W. et al.

(Korres.: gneff@tgh.org): Durabilty of rifaximin response in hepatic encephalopathy. J Clin Gastroenterol 46 (2012) 168-171

\section{Achtung:}

\section{Hier muss der Dummy durch eine Anzeige nrontzt isinrdon II}

